\title{
Estimates and Existence Results for some Fully Nonlinear Elliptic Equations on Riemannian Manifolds
}

\author{
JEFF A. VIACLOVSKY
}

\section{Introduction.}

We examine the following fully nonlinear partial differential equation on a smooth compact $n$-dimensional Riemannian manifold $(N, g)$

$$
\sigma_{k}^{1 / k}\left(\nabla^{2} u+d u \otimes d u-\frac{|\nabla u|^{2}}{2} g+S\right)=\psi(x, u)>0,
$$

where $\sigma_{k}$ is the $k$ th elementary symmetric function of the eigenvalues, $S$ is a symmetric tensor, $\nabla$ denotes the gradient, $\nabla^{2}$ denotes the Hessian, and $d u$ is the differential of $u$.

Definition 1. Let $\left(\lambda_{1}, \ldots, \lambda_{n}\right) \in \mathbf{R}^{n}$. We view the elementary symmetric functions as functions on $\mathbf{R}^{n}$

$$
\sigma_{k}\left(\lambda_{1}, \ldots, \lambda_{n}\right)=\sum_{i_{1}<\cdots<i_{k}} \lambda_{i_{1}} \cdots \lambda_{i_{k}}
$$

and we define

$$
\Gamma_{k}^{+}=\text {component of }\left\{\sigma_{k}>0\right\} \text { containing the positive cone. }
$$

We also define $\Gamma_{k}^{-}=-\Gamma_{k}^{+}$.

For a symmetric linear transformation $A: V \rightarrow V$, where $V$ is an $n$ dimensional inner product space, the notation $A \in \Gamma_{k}^{ \pm}$will mean that the eigenvalues of $A$ lie in the corresponding set. We note that this notation also makes sense for a symmetric tensor on a Riemannian manifold.

We assume the following conditions

$$
S \in \Gamma_{k}^{+},
$$


and there exist two constants $\underline{\delta}<0<\bar{\delta}$ with

$$
\psi(x, \underline{\delta})<\sigma_{k}^{1 / k}(S)<\psi(x, \bar{\delta}) \text { for all } x \in N .
$$

For example, we may take $S=g$, and $\psi(x, u)=f(x) e^{u}$, with $f(x)>0$ any smooth positive function. We shall see that (1.2) is the condition for ellipticity, and (1.3) is the $C^{0}$ estimate. For equation (1.1) with $1 \leq k \leq n$, we will prove

Theorem 1. If $S \in C^{\infty}, \psi \in C^{\infty}$, and both (1.2) and (1.3) are satisfied, then there exists at least one solution $u \in C^{\infty}(N)$ to (1.1) satisfying $\underline{\delta}<$ $u<\bar{\delta}$.

In the beautiful paper, [Li90], Yanyan Li proves the existence of a solution to the following equation on a compact Riemannian manifold

$$
\sigma_{k}^{1 / k}\left(\nabla^{2} u+I\right)=\psi(x, u)>0,
$$

provided that $N$ has non-negative sectional curvature. We would like to emphasize that because of the quadratic gradient terms in equation (1.1), we do not require any curvature assumption in our existence theorem.

The main part of our proof is the derivation of an a priori $C^{2}$ estimate on solutions. The $C^{2, \alpha}$ estimate follows from the work of Evans [Eva82], and Krylov [Kry83] for concave, uniformly elliptic equations. See also [GT83] for an excellent exposition of these results. From these estimates, we obtain the existence theorem by applying the degree theory for fully nonlinear second order elliptic equations developed by Yanyan $\mathrm{Li}$ in [Li89].

We will also discuss the equations (1.1), when $S \in \Gamma_{k}^{-}$, the negative cone. By sending $u$ to $-u$, we see that the negative case is equivalent to the positive cone case of the following equation

$$
\sigma_{k}^{1 / k}\left(\nabla^{2} u-d u \otimes d u+\frac{|\nabla u|^{2}}{2} g+S\right)=\psi(x, u)>0 .
$$

In Section 7, we will show for $\psi(x, u)=f(x) e^{u}$, the $C^{1}$ estimate still holds for this equation, but our method for obtaining the $C^{2}$ estimate does not work. We do not know if there exists a solution in this case.

\subsection{Conformal Geometry.}

We would also like to point out that (1.1) has geometric origin in conformal geometry; see [Via00a]. Let $(N, g)$ be a Riemannian manifold of dimension 
$n \geq 3$, and we define

$$
A_{g} \equiv \frac{1}{n-2}\left(R i c-\frac{R}{2(n-1)} g\right),
$$

where Ric and $R$ are the Ricci tensor and scalar curvature of the metric $g$, respectively. We consider the curvature equation

$$
\sigma_{k}^{1 / k}\left(A_{\tilde{g}}\right)=\text { constant }>0,
$$

for metrics $\tilde{g}$ in the conformal class of $g$. Notice that for $k=1$, the trace, this is just the Yamabe equation.

If we let $\tilde{g}=e^{-2 u} g$, then the curvature equation (1.5) may be written as the partial differential equation (see [Via00c])

$$
\sigma_{k}^{1 / k}\left(\nabla^{2} u+d u \otimes d u-\frac{|\nabla u|^{2}}{2} g+A_{g}\right)=e^{-2 u},
$$

where we have normalized the constant to be 1 . This equation is conformally invariant; see [Via00b].

If $A_{g} \in \Gamma_{k}^{+}$, the equation (1.6) does not satisfy (1.3), but our results here reduce the compactness question to obtaining a $C^{0}$ estimate on solutions. To this end, for the determinant case, we have the following. Let $\Omega=\{\tilde{g} \in$ $\left.[g]: A_{\tilde{g}} \in \Gamma_{n}^{+}\right\}$, where $[g]$ denotes the conformal class of $g$, and define the conformal invariant

$$
\sigma([g])=\inf _{\tilde{g} \in \Omega}\left(\lambda_{\max }\left(A_{\tilde{g}}\right) D^{2}\right),
$$

where $\lambda_{\max }\left(A_{\tilde{g}}\right)$ denotes the maximum eigenvalue of the curvature $A_{\tilde{g}}$ on $N$, and $D$ is the diameter of $(N, \tilde{g})$. If $\Omega$ is empty, then define $\sigma([g])=\infty$.

Theorem 2. If $(N,[g])$ satisifes $\sigma([g])<\frac{\pi^{2}}{2}$, then there exists $\tilde{g} \in[g]$ satisfying

$$
\operatorname{det}\left(A_{\tilde{g}}\right)=1 .
$$

Furthermore, the space of solutions of (1.8) is compact.

In Section 8 will show that, in this case, convexity yields a Harnack inequality for solutions which, together with a maximum principle argument, produces the necessary $C^{0}$ estimate. To show existence, we use a fixed point theorem of Berger ([Ber77]), following an argument from the paper of 
Delanoë [Del81]. We will also give some examples of manifolds satisfying the condition $\sigma([g])<\frac{\pi^{2}}{2}$, and demonstrate that $\sigma\left(S^{n},\left[g_{0}\right]\right)=\frac{\pi^{2}}{2}$, where $\left(S^{n}, g_{0}\right)$ is the $n$-sphere with the standard metric. Therefore Theorem 2 is analogous to the first step in the solution of the Yamabe problem: if the $\sigma$-invariant is strictly less than that of the sphere, one has existence of solutions and compactness of the space of solutions.

The case $k=1$, the Yamabe Problem, has been solved by Aubin and Schoen (see [LP87], [Sch89]), and the proof of the $C^{0}$ estimate for the Yamabe equation in the locally conformally flat case, along with an brief outline of the proof in the general case, may be found in [Sch91]. Because of the conformal invariance of equation (1.6), it is reasonable to expect that we also have compactness for all $k, 1 \leq k \leq n$, if $(N, g)$ is not conformally equivalent to $\left(S^{n}, g_{0}\right)$ :

Conjecture 1. If $A_{g} \in \Gamma_{k}^{+}$, then there exists a conformal deformation $\tilde{g}=e^{2 u} g$ such that $\sigma_{k}\left(A_{\tilde{g}}\right)=1$. Furthermore, if $(N, g)$ is not conformally equivalent to $S^{n}$ with the standard metric, then the space of solutions is compact.

Again, the results in this paper reduce this compactness statement of this conjecture to obtaining $C^{0}$ estimates on solutions. The existence should then follow from a suitable topological argument. We mention that recently Chang, Gursky and Yang, have proved the conjecture for $\sigma_{2}$ in dimension 4 (see [CGY01]).

Finally, if $A_{g} \in \Gamma_{k}^{-}$, then writing $\tilde{g}=e^{2 u} g$, and normalizing the constant, the equation (1.5) becomes

$$
\sigma_{k}^{1 / k}\left(\nabla^{2} u-d u \otimes d u+\frac{|\nabla u|^{2}}{2} g-A_{g}\right)=e^{2 u} .
$$

This is precisely equation (1.4) and, as mentioned above, from the results in Section 7 , we have an a priori bound on the $C^{1}$ norm of any solution. We do not know if there exists an a priori $C^{2}$ bound for solutions of this equation.

Acknowledgements. The author is especially grateful to Yanyan Li, for numerous helpful discussions on fully nonlinear equations, and to Pengfei Guan, for pointing out the improvement of the original argument of Proposition 13 which gives the best constant. He would also like to thank Alice Chang, Phillip Griffiths, Matt Gursky, Karen Uhlenbeck, Paul Yang, and Yu Yuan for their interest and many useful comments.

This material is based upon work supported under a National Science Foundation Postdoctoral Research Fellowship. 


\section{Ellipticity.}

In this section we will show that the equations (1.1) are elliptic at any solution.

Definition 2. Let $A: V \rightarrow V$ be a symmetric linear transformation where $V$ is an $n$-dimensional inner product space. For $0 \leq q \leq n$, the $q$ th Newton transformation associated with $A$ is

$$
T_{q}(A)=\sigma_{q}(A) \cdot I-\sigma_{q-1}(A) \cdot A+\cdots+(-1)^{q} A^{q} .
$$

It is proved in [Rei73] that if $A_{j}^{i}$ are the components of $A$ with respect to some basis of $V$ then

$$
T_{q}(A)_{j}^{i}=\frac{1}{q !} \delta_{j_{1} \ldots j_{q} j}^{i_{1} \ldots i_{q} i} A_{i_{1}}^{j_{1}} \cdots A_{i_{q}}^{j_{q}}
$$

where $\delta_{j_{1} \ldots j_{q} j}^{i_{1} \ldots i_{q} i}$ is the generalized Kronecker delta symbol, and we are using the Einstein summation convention. We also have

$$
\sigma_{k}(A)=\frac{1}{k !} \delta_{j_{1} \ldots j_{k}}^{i_{1} \ldots i_{k}} A_{i_{1}}^{j_{1}} \cdots A_{i_{k}}^{j_{k}} .
$$

We note that if $A: \mathbf{R} \rightarrow \operatorname{Hom}(V, V)$, then

$$
\frac{d}{d t} \sigma_{k}(A(t))=T_{k-1}(A(t))_{j}^{i} \frac{d}{d t} A(t)_{i}^{j}=T_{k-1}(A(t))^{i j} \frac{d}{d t} A(t)_{i j},
$$

that is, the $(k-1)$-Newton transformation is what we get when we differentiate $\sigma_{k}$.

The following proposition describes some important properties of the sets $\Gamma_{k}^{+}$.

Proposition 1. Each set $\Gamma_{k}^{+}$is an open convex cone with vertex at the origin, and we have the following sequence of inclusions

$$
\Gamma_{n}^{+} \subset \Gamma_{n-1}^{+} \subset \cdots \subset \Gamma_{1}^{+} .
$$

For symmetric linear transformations $A \in \Gamma_{k}^{+}, B \in \Gamma_{k}^{+}$, and $t \in[0,1]$, we have the following inequality

$$
\left\{\sigma_{k}((1-t) A+t B)\right\}^{1 / k} \geq(1-t)\left\{\sigma_{k}(A)\right\}^{1 / k}+t\left\{\sigma_{k}(B)\right\}^{1 / k} .
$$

Furthermore, if $A \in \Gamma_{k}^{+}$, then $T_{k-1}(A)$ is positive definite. 
The proof of this proposition is standard, and may be found in [CNS85] and [Gär59]. Note that by replacing $A$ with $-A$, analogous statements hold for $\Gamma_{k}^{-}$. Note that the inequality (2.4) states that $\sigma_{k}^{1 / k}$ is a concave function in $\Gamma_{k}^{+}$.

Definition 3. A function $u \in C^{2}(N)$ is positive $k$-admissible, or negative $k$-admissible if

$$
\bar{\nabla}^{2} u \equiv \nabla^{2} u+d u \otimes d u-\frac{|\nabla u|^{2}}{2} g+S
$$

is everywhere in $\Gamma_{k}^{+}$or $\Gamma_{k}^{-}$, respectively.

Proposition 2. If $S \in \Gamma_{k}^{+}$, then equation (1.1) is elliptic at any solution.

Proof. Since $N$ is compact, at a minimum of the solution $u$ we have

$$
\sigma_{k}^{1 / k}\left(\nabla^{2} u(p)+S(p)\right)=\psi(p, u(p))>0,
$$

with $\nabla^{2} u$ positive semidefinite. From Proposition 1, we then have, at the minimum point, $\bar{\nabla}^{2} u$ is in $\Gamma_{k}^{+}$. Therefore since the cones are connected, by continuity we have $u$ is positive $k$-admissible. A similar argument holds in the negative $k$-admissible case.

Claim 1. If we make the conformal change of metric $\tilde{g}=e^{-2 u} g$, then for any function $h$,

$$
\nabla_{\tilde{g}}^{2}(h)=\nabla_{g}^{2}(h)+d u \otimes d h+d h \otimes d u-\langle d u, d h\rangle g .
$$

where $\nabla_{g}^{2}(h)$ is the Hessian of $h$ with respect to the metric $g$, and $\nabla_{\tilde{g}}^{2}(h)$ is taken with respect to $\tilde{g}$.

Proof. We have for the Christoffel symbols (see [Bes87])

$$
\tilde{\Gamma}_{i j}^{l}=\Gamma_{i j}^{l}-u_{i} \delta_{j}^{l}-u_{j} \delta_{i}^{l}+g_{i j} g^{l r} u_{r} .
$$

Therefore

$$
\begin{aligned}
\left(\nabla_{\tilde{g}}^{2} h\right)_{i j} & =h_{i j}-\tilde{\Gamma}_{i j}^{l} h_{l} \\
& =h_{i j}-\left(\Gamma_{i j}^{l}-u_{i} \delta_{j}^{l}-u_{j} \delta_{i}^{l}+g_{i j} g^{l r} u_{r}\right) h_{l} \\
& =\left(\nabla_{g}^{2} h\right)_{i j}+u_{i} h_{j}+u_{j} h_{i}-g^{l r} u_{r} h_{l} g_{i j} .
\end{aligned}
$$


We let

$$
F\left[u, \nabla u, \nabla^{2} u\right]=\sigma_{k}^{1 / k}\left(\nabla^{2} u+d u \otimes d u-\frac{|\nabla u|^{2}}{2} g_{0}+S\right)-\psi(x, u)
$$

From (2.3) and (2.6), we see that the linearization at the solution $u$ in the direction $h$ is given by

$$
F^{\prime}\left[u, \nabla u, \nabla^{2} u\right](h)=\sigma_{k}\left(\bar{\nabla}^{2} u\right)^{\frac{1-k}{k}} T_{k-1}\left(\bar{\nabla}^{2} u\right)^{i j}\left(\nabla_{\tilde{g}}^{2} h\right)_{i j}-\psi_{u} h
$$

Since $\bar{\nabla}^{2} u$ is in $\Gamma_{k}^{+}$, from Proposition 1 , we are done.

\section{3. $C^{0}$ estimate.}

In this section, we present the necessary $C^{0}$ estimate which will be required in the existence proof. We will give the general argument, and then also an easier argument in the case that $\psi(x, u)=f(x) e^{u}$. In order to apply the maximum principle, we need to rewrite the equation as follows. We let $w=e^{u}$, and the equations (1.1) become

$$
\sigma_{k}^{1 / k}\left(\frac{1}{w} \nabla^{2} w-\frac{1}{w^{2}} \frac{|\nabla w|^{2}}{2} g+S\right)=\psi(x, \ln w)
$$

Proposition 3. If $w_{0}$ is positive $k$-admissible, and $w_{1}$ is positive $k$ admissible, then $(1-t) w_{0}+t w_{1}$ is positive $k$-admissible for $t \in[0,1]$.

Proof. By positive $k$-admissible, we mean that the matrix

$$
\bar{\nabla}^{2} w \equiv w \nabla^{2} w-\frac{|\nabla w|^{2}}{2} g+w^{2} S
$$

is in $\Gamma_{k}^{+}$. The multiple of $w$ is irrelevant, since $w=e^{u}>0$. Letting $w_{t}(x)=(1-t) w_{0}(x)+t w_{1}(x)$, we must show that $\bar{\nabla}^{2} w_{t} \in \Gamma_{k}^{+}$, i.e., $F_{k}$ is 
elliptic at $w_{t}$ for $t \in[0,1]$. We have

$$
\begin{aligned}
\bar{\nabla}^{2} w_{t}= & w_{t} \nabla^{2} w_{t}-\frac{\left|\nabla w_{t}\right|^{2}}{2} g+\left((1-t) w_{0}+t w_{1}\right)^{2} S \\
= & \left((1-t) w_{0}+t w_{1}\right)\left((1-t) \nabla^{2} w_{0}+t \nabla^{2} w_{1}\right) \\
& -\frac{\left|\nabla\left((1-t) w_{0}+t w_{1}\right)\right|^{2}}{2} g \\
& +(1-t)^{2} w_{0}^{2} S+2 t(1-t) w_{0} w_{1} S+t^{2} w_{1}^{2} S \\
= & (1-t)^{2} w_{0} \nabla^{2} w_{0}+t^{2} w_{1} \nabla^{2} w_{1}+t(1-t)\left(w_{0} \nabla^{2} w_{1}+w_{1} \nabla^{2} w_{0}\right) \\
& -\left((1-t)^{2} \frac{\left|\nabla w_{0}\right|^{2}}{2}+t(1-t) \nabla w_{1} \cdot \nabla w_{0}+t^{2} \frac{\left|\nabla w_{1}\right|^{2}}{2}\right) g \\
& +(1-t)^{2} w_{0}^{2} S+2 t(1-t) w_{0} w_{1} S+t^{2} w_{1}^{2} S \\
= & (1-t)^{2} \bar{\nabla}^{2} w_{0}+t^{2} \bar{\nabla}^{2} w_{1}+t(1-t) \\
& \cdot\left(\frac{w_{0}}{w_{1}}\left(w_{1} \nabla^{2} w_{1}-\frac{\left|\nabla w_{1}\right|^{2}}{2} g+\frac{\left|\nabla w_{1}\right|^{2}}{2} g\right)\right. \\
& \left.+\frac{w_{1}}{w_{0}}\left(w_{0} \nabla^{2} w_{0}-\frac{\left|\nabla w_{0}\right|^{2}}{2} g+\frac{\left|\nabla w_{0}\right|^{2}}{2} g\right)-\left(\nabla w_{1} \cdot \nabla w_{0}\right) g\right) \\
& +2 t(1-t) w_{0} w_{1} S \\
= & (1-t)\left((1-t) \bar{\nabla}^{2} w_{0}+t \frac{w_{1}}{w_{0}} \bar{\nabla}^{2} w_{0}\right)+t\left(t \bar{\nabla}^{2} w_{1}+(1-t) \frac{w_{0}}{w_{1}} \bar{\nabla}^{2} w_{1}\right) \\
& +\frac{t(1-t)}{2 w_{0} w_{1}}\left(w_{0}^{2}\left|\nabla w_{1}\right|^{2}+w_{1}^{2}\left|\nabla w_{0}\right|^{2}-2 w_{0} \nabla w_{1} \cdot w_{1} \nabla w_{0}\right) g \\
= & (1-t)\left((1-t) \bar{\nabla}^{2} w_{0}+t \frac{w_{1}}{w_{0}} \bar{\nabla}^{2} w_{0}\right)+t\left(t \bar{\nabla}^{2} w_{1}+(1-t) \frac{w_{1}}{w_{0}} \bar{\nabla}^{2} w_{1}\right) \\
& +\frac{t(1-t)}{2 w_{0} w_{1}}\left(\left|w_{0} \nabla w_{1}-w_{1} \nabla w_{0}\right|^{2}\right) g .
\end{aligned}
$$

From Proposition 1, the first two terms together are in $\Gamma_{k}^{+}$. The last term is a non-negative multiple of the identity, so again using Proposition 1, we are done.

Proposition 4. Suppose $S \in C^{0}, \psi \in C^{1}$, and both (1.2) and (1.3) are satisfied. Then any $C^{2}$ solution $u$ of (1.1) with $\underline{\delta} \leq u \leq \bar{\delta}$ satifies $\underline{\delta}<u<\bar{\delta}$.

Proof. Assume we have a solution $u$ of (1.1), with $\underline{\delta} \leq u$. We let

$$
F[w]=\sigma_{k}^{1 / k}\left(\frac{1}{w} \nabla^{2} w-\frac{1}{w^{2}} \frac{|\nabla w|^{2}}{2} g+S\right)-\psi(x, \ln w) .
$$


Then letting $w=e^{u}$, the function $w-e^{\underline{\delta}} \geq 0$ satisfies

$$
L\left(w-e^{\underline{\delta}}\right)=F[w]-F\left[e^{\underline{\delta}}\right]=0-\sigma_{k}^{1 / k}(S)+\psi(x, \underline{\delta})<0,
$$

where $L$ is a linear elliptic operator (this follows from Proposition 3 , see [GT83], Chapter 17), so by the maximum principle, we have $e^{\underline{\delta}}<w$, that is, $\underline{\delta}<u$. The proof of the strict upper inequality is similar.

Remark. Why did we change to $w=e^{u}$ in the above argument? A computation similar to that of the proof of Proposition 3 shows that the original equation (1.1) is elliptic along the straight line path only if $k \leq$ $n / 2$. We are just using a different straight line path in order to apply the maximum principle.

In the case that $\psi(x, u)=f(x) e^{u}$, we present an alternative, more elementary derivation of the $C^{0}$ estimate.

Lemma 1. Let $A$ and $B$ be symmetric $n \times n$ matrices. Assume that $A$ is positive semi-definite, $B \in \Gamma_{k}^{+}$, and $A+B \in \Gamma_{k}^{+}$. Then

$$
\sigma_{k}(A+B) \geq \sigma_{k}(B) \text {. }
$$

If $A$ is negative semi-definite, then

$$
\sigma_{k}(A+B) \leq \sigma_{k}(B)
$$

Proof. Let $F(t)=\sigma_{k}(t A+B)-\sigma_{k}(B)$ for $t \in[0,1]$. Note that from convexity of the cone $\Gamma_{k}^{+}$, we have $t(A+B)+(1-t) B=t A+B \in \Gamma_{k}^{+}$. Using (2.3), we have

$$
F^{\prime}(t)=T_{k-1}(t A+B)^{i j} A_{i j} \geq 0,
$$

since $T_{k-1}(t A+B)$ is positive definite from Proposition 1. Therefore $F(t)$ is non-decreasing, and $F(0)=0$, so we have $F(1)=\sigma_{k}(A+B)-\sigma_{k}(B) \geq 0$. The negative case is similar.

Proposition 5. Suppose $S \in C^{0}$ satisfies (1.2). If $\psi(x, u)=f(x) e^{u}$, for $f(x)>0$ a positive $C^{0}$ function, then there exist constants $\underline{\delta}<0<\bar{\delta}$ depending only upon $f, S$ and $k$, such that for any solution $u(x)$ of (1.1), we have $\underline{\delta}<u(x)<\bar{\delta}$. 
Proof. Since $N$ is compact, at a minimum of the function $u(x)$ we have

$$
\sigma_{k}^{1 / k}\left(\nabla^{2} u(p)+S(p)\right)=f(p) e^{u(p)}
$$

with $\nabla^{2} u(p)$ positive semidefinite. From the lemma we have

$$
\sigma_{k}^{1 / k}(S(p)) \leq f(p) e^{u(p)},
$$

and certainly we can choose $\underline{\delta}$ such that

$$
u(x) \geq u(p) \geq \ln \left(\frac{\sigma_{k}^{1 / k}(S(p))}{f(p)}\right) \geq \ln \left(\min _{x \in N} \frac{\sigma_{k}^{1 / k}(S(x))}{f(x)}\right)>\underline{\delta} .
$$

Similarly, if the maximum of $u(x)$ is at $q \in N$, we can choose $\bar{\delta}$ such that

$$
u(x) \leq u(q) \leq \ln \left(\frac{\sigma_{k}^{1 / k}(S(q))}{f(q)}\right) \leq \ln \left(\max _{x \in N} \frac{\sigma_{k}^{1 / k}(S(x))}{f(x)}\right)<\bar{\delta} .
$$

\section{4. $C^{1}$ estimate.}

Proposition 6. Suppose $S \in C^{1}, \psi \in C^{1},(1.2)$ is satisfied, and $u$ is a $C^{3}$ solution of (1.1) satisfying $\underline{\delta} \leq u(x) \leq \bar{\delta}$. Then there exists a constant $C_{1}$ depending only upon $S, \psi, \underline{\delta}, \bar{\delta}$, and $k$ such that

$$
|\nabla u|_{C^{0}} \leq C_{1} .
$$

We consider the following function

$$
h=\left(1+\frac{|\nabla u|^{2}}{2}\right) e^{\phi(u)}
$$

where $\phi: \mathbf{R} \rightarrow \mathbf{R}$ is a function of the form

$$
\phi(s)=c_{1}\left(c_{2}-s\right)^{p} .
$$

The constants $c_{1}, c_{2}$, and $p$ will be chosen later. We will estimate the maximum value of the function $h$, and this will give us the gradient estimate.

Since $N$ is compact, and $h$ is continuous, we suppose the maximum of $h$ occurs and a point $p \in N$. We take a normal coordinate system $\left(x^{1}, \ldots, x^{n}\right)$ at $p$. Then we have $g_{i j}(p)=\delta_{i j}$, and $\Gamma_{j k}^{i}(p)=0$, where $g=g_{i j} d x^{i} d x^{j}$, and $\Gamma_{j k}^{i}$ is the Christoffel symbol (see [Bes87]). 
Locally, we may write $h$ as

$$
h=\left(1+\frac{1}{2} g^{l m} u_{l} u_{m}\right) e^{\phi(u)}=v e^{\phi(u)} .
$$

In a neighborhood of $p$, differentiating $h$ in the $x^{i}$ direction we have

$$
\begin{aligned}
\partial_{i} h & =h_{i}=\frac{1}{2} \partial_{i}\left(g^{l m} u_{l} u_{m}\right) e^{\phi(u)}+v e^{\phi(u)} \phi^{\prime}(u) u_{i} \\
& =\frac{1}{2} \partial_{i}\left(g^{l m}\right) u_{l} u_{m} e^{\phi(u)}+g^{l m} \partial_{i}\left(u_{l}\right) u_{m} e^{\phi(u)}+v e^{\phi(u)} \phi^{\prime}(u) u_{i}
\end{aligned}
$$

Since in a normal coordinate system, the first derivatives of the metric vanish at $p$, and since $p$ is a maximum for $h$, evaluating (4.1) at $p$, we have

$$
u_{l i} u_{l}=-v \phi^{\prime}(u) u_{i}
$$

Next we differentiate (4.1) in the $x^{j}$ direction. Since $p$ is a maximum, $\partial_{j} \partial_{i} h=h_{i j}$ is negative semidefinite, and we get (at $p$ )

$$
\begin{aligned}
0 \gg h_{i j}= & \frac{1}{2} \partial_{j} \partial_{i} g^{l m} u_{l} u_{m} e^{\phi(u)}+u_{l i j} u_{l} e^{\phi(u)}+u_{l i} u_{l j} e^{\phi(u)} \\
& +u_{l i} u_{l} e^{\phi(u)} \phi^{\prime}(u) u_{j}+v_{j} e^{\phi(u)} \phi^{\prime}(u) u_{i}+v e^{\phi(u)}\left(\phi^{\prime}(u)\right)^{2} u_{i} u_{j} \\
& +v e^{\phi(u)} \phi^{\prime \prime}(u) u_{j} u_{i}+v e^{\phi(u)} \phi^{\prime}(u) u_{i j}
\end{aligned}
$$

Next we note that $v_{j}=u_{l j} u_{l}$, and using (4.2), we have

$$
\begin{aligned}
0 \gg h_{i j}= & \frac{1}{2} \partial_{j} \partial_{i} g^{l m} u_{l} u_{m} e^{\phi(u)}+u_{l i j} u_{l} e^{\phi(u)}+u_{l i} u_{l j} e^{\phi(u)} \\
& +\left(\phi^{\prime \prime}(u)-\phi^{\prime}(u)^{2}\right) v e^{\phi(u)} u_{i} u_{j}+v e^{\phi(u)} \phi^{\prime}(u) u_{i j}
\end{aligned}
$$

Next we divide by $v e^{\phi(u)}$, sum with $T_{k-1}\left(\bar{\nabla}^{2} u\right)^{i j}$ (which is positive definite and symmetric), and we have the inequality

$$
\begin{aligned}
0 \geq & \frac{1}{2 v} T_{k-1}^{i j} \partial_{i} \partial_{j} g^{l m} u_{l} u_{m}+\frac{1}{v} T_{k-1}^{i j} u_{l i j} u_{l} \\
& +\left(\phi^{\prime \prime}(u)-\phi^{\prime}(u)^{2}\right) T_{k-1}^{i j} u_{i} u_{j}+\phi^{\prime}(u) T_{k-1}^{i j} u_{i j}
\end{aligned}
$$

since $u_{l i} u_{l j}$ is positive semidefinite, and we abbreviate $T_{k-1}^{i j}=T_{k-1}\left(\bar{\nabla}^{2} u\right)^{i j}$, where $\bar{\nabla}^{2} u$ is the notation in (2.5) above.

We will use equation (1.1) to replace the $u_{i j}$ term with lower order terms, and then differentiate equation (1.1) in order to replace the $u_{l i j}$ term with 
lower order terms. Writing equation (1.1) with respect to our local coordinate system, we have

$$
\sigma_{k}^{1 / k}\left(g^{l j}\left(u_{i j}-u_{r} \Gamma_{i j}^{r}+u_{i} u_{j}-\frac{1}{2}\left(g^{r_{1} r_{2}} u_{r_{1}} u_{r_{2}}\right) g_{i j}+S_{i j}\right)\right)=\psi(x, u) .
$$

Note that the $g^{l j}$ term is present since we need to raise an index on the tensor before we apply $\sigma_{k}^{1 / k}$.

For a symmetric matrix $A$, we have the formula (see [Rei73])

$$
T_{k-1}(A)^{i j} A_{i j}=k \sigma_{k}(A) .
$$

Using this, and equation (4.4), we have at $p$,

$$
\begin{aligned}
T_{k-1}^{i j} u_{i j} & =T_{k-1}^{i j}\left(u_{i j}+u_{i} u_{j}-\frac{|\nabla u|^{2}}{2} \delta_{i j}+S_{i j}-u_{i} u_{j}+\frac{|\nabla u|^{2}}{2} \delta_{i j}-S_{i j}\right) \\
& =k \sigma_{k}+T_{k-1}^{i j}\left(-u_{i} u_{j}+\frac{|\nabla u|^{2}}{2} \delta_{i j}-S_{i j}\right) \\
& =k \psi(x, u)^{k}+T_{k-1}^{i j}\left(-u_{i} u_{j}+\frac{|\nabla u|^{2}}{2} \delta_{i j}-S_{i j}\right)
\end{aligned}
$$

Next we take $m$ with $1 \leq m \leq n$, and apply $\partial_{m}$ to (4.4)

$$
\begin{aligned}
& \sigma_{k}^{\frac{1-k}{k}} T_{k-1}^{i l}\left(\partial_{m} g^{l j}\left(\bar{\nabla}^{2} u\right)_{i j}+g^{l j}\left(u_{i j m}-u_{r m} \Gamma_{i j}^{r}-u_{r} \partial_{m} \Gamma_{i j}^{r}+u_{i m} u_{j}+u_{i} u_{m j}\right.\right. \\
& \left.\left.-\frac{1}{2}\left(\partial_{m} g^{r_{1} r_{2}}\right) u_{r_{1}} u_{r_{2}} g_{i j}-g^{r_{1} r_{2}} u_{r_{1} m} u_{r_{2}} g_{i j}-\frac{1}{2} g^{r_{1} r_{2}} u_{r_{1}} u_{r_{2}} \partial_{m} g_{i j}+\partial_{m} S_{i j}\right)\right) \\
& \quad=\frac{\partial \psi}{\partial x^{m}}+\frac{\partial \psi}{\partial u} u_{m} .
\end{aligned}
$$

We evaluate the above expression at $p$, and we obtain

$$
\psi^{1-k} T_{k-1}^{i j}\left(u_{i j m}-u_{r} \partial_{m} \Gamma_{i j}^{r}+2 u_{i m} u_{j}-u_{r m} u_{r} \delta_{i j}+\partial_{m} S_{i j}\right)=\psi_{m}+\psi_{u} u_{m} .
$$

We then sum with $u_{m}$, and using (4.2) we have the following formula

$$
\begin{aligned}
& T_{k-1}^{i j} u_{i j m} u_{m} \\
& =T_{k-1}^{i j}\left(u_{m} u_{r} \partial_{m} \Gamma_{i j}^{r}+2 v \phi^{\prime}(u) u_{i} u_{j}-v \phi^{\prime}(u)|\nabla u|^{2} \delta_{i j}+u_{m} \partial_{m} S_{i j}\right) \\
& \quad+\psi^{k-1}\left(u_{m} \psi_{m}+\psi_{u}|\nabla u|^{2}\right) .
\end{aligned}
$$


Substituting (4.5) and (4.8) into (4.3), we arrive at the inequality

$$
\begin{aligned}
& 0 \geq \frac{1}{2 v} T_{k-1}^{i j} \partial_{i} \partial_{j} g^{l m} u_{l} u_{m} \\
&+\frac{1}{v} T_{k-1}^{i j}\left(u_{l} u_{r} \partial_{l} \Gamma_{i j}^{r}+2 v \phi^{\prime}(u) u_{i} u_{j}-v \phi^{\prime}(u)|\nabla u|^{2} \delta_{i j}+u_{l} \partial_{l} S_{i j}\right) \\
&+\frac{\psi^{k-1}}{v}\left(u_{m} \psi_{m}+\psi_{u}|\nabla u|^{2}\right)+\left(\phi^{\prime \prime}(u)-\phi^{\prime}(u)^{2}\right) T_{k-1}^{i j} u_{i} u_{j} \\
&(4.9) \quad+k \phi^{\prime}(u) \psi(x, u)^{k}+\phi^{\prime}(u) T_{k-1}^{i j}\left(-u_{i} u_{j}+\frac{|\nabla u|^{2}}{2} \delta_{i j}-S_{i j}\right)
\end{aligned}
$$

Lemma 2. At $p$, in normal coordinates, we have

$$
\sum_{l, m}\left(\partial_{i} \partial_{j} g^{l m}+2 \partial_{l} \Gamma_{i j}^{m}\right) u_{l} u_{m}=2 \sum_{l, m} R_{i l j m} u_{l} u_{m}
$$

where $R_{i l j m}$ are the components of the Riemann curvature tensor of $g$ (see [Bes87]).

Proof. The metric is parallel, so we have

$$
0=\nabla_{j} g^{l m}=\partial_{j} g^{l m}+\Gamma_{j r}^{l} g^{r m}+\Gamma_{j r}^{m} g^{l r} .
$$

Therefore we have, at $p$,

$$
0=\partial_{i} \partial_{j} g^{l m}+\partial_{i} \Gamma_{j r}^{l} \delta^{r m}+\partial_{i} \Gamma_{j r}^{m} \delta^{l r}=\partial_{i} \partial_{j} g^{l m}+\partial_{i} \Gamma_{j m}^{l}+\partial_{i} \Gamma_{j l}^{m}
$$

Using this, we have

$$
\begin{aligned}
\sum_{l, m}\left(\partial_{i} \partial_{j} g^{l m}+2 \partial_{l} \Gamma_{i j}^{m}\right) u_{l} u_{m} & =\sum_{l, m}\left(-\partial_{i} \Gamma_{j m}^{l}-\partial_{i} \Gamma_{j l}^{m}+2 \partial_{l} \Gamma_{i j}^{m}\right) u_{l} u_{m} \\
& =2 \sum_{l, m}\left(-\partial_{i} \Gamma_{l j}^{m}+\partial_{l} \Gamma_{i j}^{m}\right) u_{l} u_{m} \\
& =2 \sum_{l, m} R_{i l j m} u_{l} u_{m} .
\end{aligned}
$$


Using the lemma, and collecting terms in (4.9), we arrive at

$$
\begin{aligned}
& -\psi^{k-1}\left(\frac{u_{m}}{v} \psi_{m}+\psi_{u} \frac{|\nabla u|^{2}}{v}\right)-k \phi^{\prime}(u) \psi(x, u)^{k} \\
& \geq\left(\phi^{\prime \prime}(u)-\phi^{\prime}(u)^{2}+\phi^{\prime}(u)\right) T_{k-1}^{i j} u_{i} u_{j} \\
& \quad+T_{k-1}^{i j}\left(R_{i l j m} \frac{u_{l} u_{m}}{v}-\phi^{\prime}(u) \frac{|\nabla u|^{2}}{2} \delta_{i j}-\phi^{\prime}(u) S_{i j}+\frac{u_{l}}{v} \partial_{l} S_{i j}\right) .
\end{aligned}
$$

Now we will choose $\phi(s)$.

Lemma 3. Assume that $\underline{\delta}<s<\bar{\delta}$. Then we may choose constants $c_{1}, c_{2}$, and $p$ depending only upon $\underline{\delta}$, and $\bar{\delta}$. so that $\phi(s)=c_{1}\left(c_{2}-s\right)^{p}$ satisfies

$$
\phi^{\prime}(s)<0
$$

and

$$
\phi^{\prime \prime}(s)-\phi^{\prime}(s)^{2}+\phi^{\prime}(s)>0
$$

Proof. We have

$$
\phi^{\prime}(s)=-p c_{1}\left(c_{2}-s\right)^{p-1}
$$

and

$$
\phi^{\prime \prime}(s)=p(p-1) c_{1}\left(c_{2}-s\right)^{p-2} .
$$

To satisfy (4.11) we need $c_{1}>0, p>0$, and $c_{2}>s$. So choose $c_{2}>\bar{\delta}$. Next we have

$$
\begin{aligned}
& \phi^{\prime \prime}(s)-\phi^{\prime}(s)^{2}+\phi^{\prime}(s) \\
& =p(p-1) c_{1}\left(c_{2}-s\right)^{p-2}-\left(p c_{1}\left(c_{2}-s\right)^{p-1}\right)^{2}-p c_{1}\left(c_{2}-s\right)^{p-1} \\
& =p c_{1}\left(c_{2}-s\right)^{p-2}\left((p-1)-p c_{1}\left(c_{2}-s\right)^{p}-\left(c_{2}-s\right)\right) .
\end{aligned}
$$

Now choose

$$
c_{1}=\frac{1}{p^{2} \cdot \max \left\{\left(c_{2}-s\right)^{p}\right\}}
$$

and $p$ so large that

$$
\bar{\delta}<c_{2}<\underline{\delta}+p-1-\frac{1}{p}
$$


Then we have

$$
\begin{aligned}
\phi^{\prime \prime}(s)-\phi^{\prime}(s)^{2}+\phi^{\prime}(s) & \geq \frac{1}{p \cdot \max \left\{\left(c_{2}-s\right)^{p}\right\}}\left(c_{2}-s\right)^{p-2}\left(p-1-\frac{1}{p}-c_{2}+s\right) \\
& >\frac{1}{p \cdot \max \left\{\left(c_{2}-s\right)^{p}\right\}}\left(c_{2}-s\right)^{p-2}(-\underline{\delta}+s)>0 .
\end{aligned}
$$

With $\phi(s)$ chosen as above, we let

$$
\epsilon_{1}=-\max \left\{\phi^{\prime}(s)\right\}
$$

and

$$
\epsilon_{2}=\min \left\{\phi^{\prime \prime}(s)-\phi^{\prime}(s)^{2}+\phi^{\prime}(s)\right\} .
$$

From the inequality (4.10), we have

$$
C \geq \epsilon_{2} T_{k-1}^{i j} u_{i} u_{j}+T_{k-1}^{i j}\left(R_{i l j m} \frac{u_{l} u_{m}}{v}+\epsilon_{1} \frac{|\nabla u|^{2}}{2} \delta_{i j}+\phi^{\prime}(u) S_{i j}+\frac{u_{l}}{v} \partial_{l} S_{i j}\right),
$$

where in this equation, and in what follows, $C$ is a constant depending on $\bar{\delta}, \underline{\delta}$, and $\psi$.

Without loss of generality, assume that $\bar{\nabla}^{2} u$ is diagonal at $p$. Now if for some $i$, a diagonal entry of the matrix in parenthesis above satisfies

$$
R_{i l i m} \frac{u_{l} u_{m}}{v}+\epsilon_{1} \frac{|\nabla u|^{2}}{2}+\phi^{\prime}(u) S_{i i}+\frac{u_{l}}{v} \partial_{l} S_{i i}<1,
$$

then we have the gradient bound. So we may assume that

$$
R_{i l i m} \frac{u_{l} u_{m}}{v}+\epsilon_{1} \frac{|\nabla u|^{2}}{2}+\phi^{\prime}(u) S_{i i}+\frac{u_{l}}{v} \partial_{l} S_{i i} \geq 1
$$

for all $i$. From the inequality (4.13), we conclude that

$$
C \geq \epsilon_{2} \sum_{i} T_{k-1}^{i i} u_{i}^{2}+\sum_{i} T_{k-1}^{i i}
$$

Noting that

$$
\sum_{i} T_{k-1}^{i i}=(n-k+1) \sigma_{k-1}
$$

(see [Rei73]) we deduce that

$$
\sigma_{k-1} \leq C
$$


Proposition 7. Let $k \geq 2$, and $A \in \Gamma_{k}^{+}$be a symmetric linear transformation. If $0<c_{1} \leq \sigma_{k}(A)$, and $\sigma_{k-1}(A) \leq c_{2}$, then we have a bound on the eigenvalues of $A$, that is, $|\lambda(A)| \leq C$, where $C$ depends only on $c_{1}$ and $c_{2}$.

Proof. The proof may be found in [Li90].

Using this result, if $k \geq 2$, we see that

$$
|\lambda| \leq C,
$$

and since $T_{k-1}$ is positive definite, this implies

$$
T_{k-1}^{i i} \geq \frac{1}{C}>0, \text { for } i=1 \ldots n \text {. }
$$

Equation (4.14) then implies that

$$
|\nabla u|^{2} \leq C
$$

Note that in the case $k=1$, we do not require the proposition since $T_{0}^{i j}=\delta^{i j}$, and therefore (4.14) gives the gradient bound.

\section{5. $C^{2}$ estimate.}

Proposition 8. Suppose $S \in C^{2}, \psi \in C^{2}$, (1.2) is satisfied, $u$ is a $C^{4}$ solution of (1.1) satisfying $\underline{\delta} \leq u(x) \leq \bar{\delta}$, and $|\nabla u|<C_{1}$. Then there exists a constant $C_{2}$ depending only upon $S, \psi, \underline{\delta}, \bar{\delta}, C_{1}$, and $k$ such that

$$
\left|\nabla^{2} u\right|_{C^{0}} \leq C_{2}
$$

Let $S(T N)$ denote the unit tangent bundle of $N$, and we consider the following function $w: S(T N) \mapsto \mathbf{R}$,

$$
w\left(e_{p}\right)=\left(\nabla^{2} u+d u \otimes d u+S\right)\left(e_{p}, e_{p}\right) .
$$

Since $S(T N)$ is compact, let $w$ have a maximum at the vector $\tilde{e}_{p}$. We use normal coordinates at $p$, and by rotating, assume that the tensor is diagonal at $p$, and without loss of generality, we may assume that $\tilde{e}_{p}=\partial / \partial x^{1}$.

We let $\tilde{w}$ denote the function defined in a neighborhood of $p$

$$
\begin{aligned}
\tilde{w}(x) & =\left(\nabla^{2} u+d u \otimes d u+S\right)\left(\partial / \partial x^{1}, \partial / \partial x^{1}\right) \\
& =\left(\nabla^{2} u\right)_{11}+u_{1}^{2}+S_{11} \\
& =u_{11}-\Gamma_{11}^{l} u_{l}+u_{1}^{2}+S_{11} .
\end{aligned}
$$


Differentiating in the $i$ th coordinate direction, we obtain

$$
\tilde{w}_{i}=u_{11 i}-\partial_{i} \Gamma_{11}^{l} u_{l}-\Gamma_{11}^{l} u_{l i}+2 u_{1} u_{1 i}+\partial_{i} S_{11} .
$$

The function $\tilde{w}(x)$ has a maximum at $p$, so evaluating (5.1) at $p$, we obtain

$$
u_{11 i}=\partial_{i} \Gamma_{11}^{l} u_{l}-2 u_{1} u_{1 i}-\partial_{i} S_{11} .
$$

Next we differentiate (5.1) in the $x^{j}$ direction. Since $p$ is a maximum, $\partial_{j} \partial_{i} \tilde{w}=\tilde{w}_{i j}$ is negative semidefinite, and we get (at $p$ )

$$
\begin{aligned}
0 \gg \tilde{w}_{i j}= & u_{11 i j}-\partial_{i} \partial_{j} \Gamma_{11}^{l} u_{l}-\partial_{i} \Gamma_{11}^{l} u_{l j}-\partial_{j} \Gamma_{11}^{l} u_{l i} \\
& +2 u_{1 j} u_{1 i}+2 u_{1} u_{1 i j}+\partial_{i} \partial_{j} S_{11} .
\end{aligned}
$$

We sum with $T_{k-1}\left(\bar{\nabla}^{2} u\right)^{i j}$ (which is positive definite and symmetric), and we have the inequality

$$
\begin{aligned}
0 \geq & T_{k-1}^{i j} u_{11 i j}-T_{k-1}^{i j} \partial_{i} \partial_{j} \Gamma_{11}^{l} u_{l}-2 T_{k-1}^{i j} \partial_{i} \Gamma_{11}^{l} u_{l j} \\
& +2 T_{k-1}^{i j} u_{1 j} u_{1 i}+2 T_{k-1}^{i j} u_{1} u_{1 i j}+T_{k-1}^{i j} \partial_{i} \partial_{j} S_{11} .
\end{aligned}
$$

We will use (4.7) to replace the fifth term, and we will differentiate equation (1.1) twice to replace the first term.

We recall that the equation is

$$
\sigma_{k}^{1 / k}\left(\bar{\nabla}^{2} u\right)=\psi(x, u)
$$

To simplify notation, write $f=\sigma_{k}^{1 / k}$. Differentiating once in the $x^{1}$ direction, we had (equation (4.6))

$$
\frac{\partial f}{\partial r_{i j}}\left(\partial_{1}\left(\bar{\nabla}^{2} u\right)_{j}^{i}\right)=\psi_{1}+\psi_{u} u_{1}
$$

Differentiating twice, we obtain

$$
\begin{aligned}
& \partial_{1}\left(\frac{\partial f}{\partial r_{i j}}\right)\left(\partial_{1}\left(\bar{\nabla}^{2} u\right)_{j}^{i}\right)+\frac{\partial f}{\partial r_{i j}}\left(\partial_{1} \partial_{1}\left(\bar{\nabla}^{2} u\right)_{j}^{i}\right) \\
& =\left(\frac{\partial^{2} f}{\partial r_{i j} \partial r_{l m}}\right)\left(\partial_{1}\left(\bar{\nabla}^{2} u\right)_{m}^{l}\right)\left(\partial_{1}\left(\bar{\nabla}^{2} u\right)_{j}^{i}\right)+\frac{\partial f}{\partial r_{i j}}\left(\partial_{1} \partial_{1}\left(\bar{\nabla}^{2} u\right)_{j}^{i}\right) \\
& =\psi_{11}+2 \psi_{1 u} u_{1}+\psi_{u u} u_{1}^{2}+\psi_{u} u_{11} .
\end{aligned}
$$

Since $\sigma_{k}^{1 / k}$ is concave in $\Gamma_{k}^{+}$, we have the inequality

$$
T_{k-1}^{i j}\left(\partial_{1} \partial_{1}\left(\bar{\nabla}^{2} u\right)_{j}^{i}\right) \geq \psi^{k-1}\left(\psi_{11}+2 \psi_{1 u} u_{1}+\psi_{u u} u_{1}^{2}+\psi_{u} u_{11}\right) .
$$


From formula (4.6), we can expand the left hand side, and evaluate at $p$ to get

$$
\begin{aligned}
& T_{k-1}^{i j}\left(\partial_{1} \partial_{1}\left(\bar{\nabla}^{2} u\right)_{j}^{i}\right) \\
& =T_{k-1}^{i j}\left(\partial_{1} \partial_{1} g^{j l}\left(\bar{\nabla}^{2} u\right)_{i l}\right)+T_{k-1}^{i j}\left(u_{i j 11}-2 u_{r 1} \partial_{1} \Gamma_{i j}^{r}-u_{r} \partial_{1} \partial_{1} \Gamma_{i j}^{r}\right. \\
& \quad+2 u_{j} u_{i 11}+2 u_{i 1} u_{j 1}-\frac{1}{2} \partial_{1} \partial_{1} g^{r_{1} r_{2}} u_{r_{1}} u_{r_{2}} \delta_{i j}-\left(u_{r 11} u_{r}+u_{r 1} u_{r 1}\right) \delta_{i j} \\
& \left.\quad-\frac{1}{2}|\nabla u|^{2} \partial_{1} \partial_{1}\left(g_{i j}\right)+\partial_{1} \partial_{1} S_{i j}\right) .
\end{aligned}
$$

From (5.2) we can replace terms of the form $u_{11 i}$ and we have

$$
\begin{aligned}
& T_{k-1}^{i j}\left(\partial_{1} \partial_{1}\left(\bar{\nabla}^{2} u\right)_{j}^{i}\right) \\
& =T_{k-1}^{i j}\left(\partial_{1} \partial_{1} g^{j l}\left(\bar{\nabla}^{2} u\right)_{i l}\right)+T_{k-1}^{i j}\left(u_{i j 11}-2 u_{r 1} \partial_{1} \Gamma_{i j}^{r}-u_{r} \partial_{1} \partial_{1} \Gamma_{i j}^{r}\right. \\
& \quad+2 u_{j}\left(\partial_{i} \Gamma_{11}^{l} u_{l}-2 u_{1} u_{1 i}-\partial_{i} S_{11}\right)+2 u_{i 1} u_{j 1}-\frac{1}{2} \partial_{1} \partial_{1} g^{r_{1} r_{2}} u_{r_{1}} u_{r_{2}} \delta_{i j} \\
& \quad-\left(u_{r}\left(\partial_{r} \Gamma_{11}^{l} u_{l}-2 u_{1} u_{1 r}-\partial_{r} S_{11}\right)+u_{r 1} u_{r 1}\right) \delta_{i j} \\
& \left.\quad-\frac{1}{2}|\nabla u|^{2} \partial_{1} \partial_{1}\left(g_{i j}\right)+\partial_{1} \partial_{1} S_{i j}\right) .
\end{aligned}
$$

Substituting (5.5) in (5.4), we have

$$
\begin{aligned}
T_{k-1}^{i j} u_{i j 11} \geq- & T_{k-1}^{i j}\left(\partial_{1} \partial_{1} g^{j l}\left(\bar{\nabla}^{2} u\right)_{i l}\right) \\
& +T_{k-1}^{i j}\left(2 u_{r 1} \partial_{1} \Gamma_{i j}^{r}+u_{r} \partial_{1} \partial_{1} \Gamma_{i j}^{r}-2 u_{i 1} u_{j 1}\right) \\
& +2 T_{k-1}^{i j}\left(-u_{j} \partial_{i} \Gamma_{11}^{l} u_{l}+2 u_{j} u_{1} u_{1 i}+u_{j} \partial_{i} S_{11}\right) \\
& +T_{k-1}^{i j}\left(u_{r} \partial_{r} \Gamma_{11}^{l} u_{l}-2 u_{1} u_{r} u_{1 r}-u_{r} \partial_{r} S_{11}+u_{r 1} u_{r 1}\right) \delta_{i j} \\
& +T_{k-1}^{i j}\left(\frac{1}{2} \partial_{1} \partial_{1} g^{r_{1} r_{2}} u_{r_{1}} u_{r_{2}} \delta_{i j}+\frac{1}{2}|\nabla u|^{2} \partial_{1} \partial_{1} g_{i j}-\partial_{1} \partial_{1} S_{i j}\right) \\
& +\psi^{k-1}\left(\psi_{11}+2 \psi_{1 u} u_{1}+\psi_{u u} u_{1}^{2}+\psi_{u} u_{11}\right) .
\end{aligned}
$$

Next we will substitute inequality (5.6) into (5.3). Note that the fourth term on the right hand side of (5.6) will cancel the fourth term in (5.3). We also 
use equation (4.7) to replace the fifth term in (5.3). We have

$$
\begin{aligned}
0 \geq- & T_{k-1}^{i j}\left(\partial_{1} \partial_{1} g^{j l}\left(\bar{\nabla}^{2} u\right)_{i l}\right)+T_{k-1}^{i j}\left(2 u_{r 1} \partial_{1} \Gamma_{i j}^{r}+u_{r} \partial_{1} \partial_{1} \Gamma_{i j}^{r}\right) \\
& +2 T_{k-1}^{i j}\left(-u_{j} \partial_{i} \Gamma_{11}^{l} u_{l}+2 u_{j} u_{1} u_{1 i}+u_{j} \partial_{i} S_{11}\right) \\
& +T_{k-1}^{i j}\left(u_{r} \partial_{r} \Gamma_{11}^{l} u_{l}-2 u_{1} u_{r} u_{1 r}-u_{r} \partial_{r} S_{11}+u_{r 1} u_{r 1}\right) \delta_{i j} \\
& +T_{k-1}^{i j}\left(\frac{1}{2} \partial_{1} \partial_{1} g^{r_{1} r_{2}} u_{r_{1}} u_{r_{2}} \delta_{i j}+\frac{1}{2}|\nabla u|^{2} \partial_{1} \partial_{1} g_{i j}-\partial_{1} \partial_{1} S_{i j}\right) \\
& +\psi^{k-1}\left(\psi_{11}+2 \psi_{1 u} u_{1}+\psi_{u u} u_{1}^{2}+\psi_{u} u_{11}\right) \\
& -T_{k-1}^{i j} \partial_{i} \partial_{j} \Gamma_{11}^{l} u_{l}-2 T_{k-1}^{i j} \partial_{i} \Gamma_{11}^{l} u_{l j} \\
& +2 T_{k-1}^{i j}\left(u_{1} u_{r} \partial_{1} \Gamma_{i j}^{r}-2 u_{1} u_{i 1} u_{j}+u_{1} u_{r 1} u_{r} \delta_{i j}-u_{1} \partial_{1} S_{i j}\right) \\
& +2 \psi^{k-1}\left(\psi_{1} u_{1}+\psi_{u} u_{1}^{2}\right)+T_{k-1}^{i j} \partial_{i} \partial_{j} S_{11}
\end{aligned}
$$

Note that the boxed terms cancel. Using the bounds on lower order quantities, the above simplifies to

$$
\begin{aligned}
C+C \sum_{i} T_{k-1}^{i i} & \geq-T_{k-1}^{i j}\left(\partial_{1} \partial_{1} g^{j l}\left(\bar{\nabla}^{2} u\right)_{i l}\right)+2 T_{k-1}^{i j}\left(u_{r 1} \partial_{1} \Gamma_{i j}^{r}\right) \\
& +T_{k-1}^{i j} u_{r 1} u_{r 1} \delta_{i j}+\psi^{k-1} \psi_{u} u_{11}-2 T_{k-1}^{i j} \partial_{i} \Gamma_{11}^{l} u_{l j} .
\end{aligned}
$$

In this equation, and in what follows, $C$ is a constant depending only on $S, \psi, \underline{\delta}, \bar{\delta}, C_{1}$, and $k$.

The next step is to rewrite the second derivative terms in terms of $\bar{\nabla}^{2} u$. To further simplify notation, we let $\bar{u}_{i j}=\left(\bar{\nabla}^{2} u\right)_{i j}$. We have

$$
u_{i j}=\bar{u}_{i j}-u_{i} u_{j}+\left(|\nabla u|^{2} / 2\right) \delta_{i j}-S_{i j} .
$$

Substituting this into (5.7), we obtain

$$
\begin{aligned}
C+C \sum_{i} T_{k-1}^{i i} & \geq-T_{k-1}^{i j}\left(\partial_{1} \partial_{1} g^{j l} \bar{u}_{i l}\right) \\
& +2 T_{k-1}^{i j}\left(\bar{u}_{r 1}-u_{r} u_{1}+\left(|\nabla u|^{2} / 2\right) \delta_{r 1}-S_{r 1}\right) \partial_{1} \Gamma_{i j}^{r} \\
& +\left(\sum_{i} T_{k-1}^{i i}\right) \sum_{r}\left(\bar{u}_{r 1}-u_{r} u_{1}+\left(|\nabla u|^{2} / 2\right) \delta_{r 1}-S_{r 1}\right)^{2} \\
& +\psi^{k-1} \psi_{u}\left(\bar{u}_{11}-u_{1}^{2}+\left(|\nabla u|^{2} / 2\right)-S_{11}\right) \\
& -2 T_{k-1}^{i j} \partial_{i} \Gamma_{11}^{l}\left(\bar{u}_{l j}-u_{l} u_{j}+\left(|\nabla u|^{2} / 2\right) \delta_{l j}-S_{l j}\right) .
\end{aligned}
$$


Next we use the fact that $\bar{u}_{i j}$ is diagonal, and absorbing lower order terms we obtain

$$
\begin{aligned}
C & +C \sum_{i} T_{k-1}^{i i}+C \bar{u}_{11} \sum_{i} T_{k-1}^{i i} \\
\geq & -\sum_{i} T_{k-1}^{i i}\left(\partial_{1} \partial_{1} g^{i i} \bar{u}_{i i}\right)-2 \sum_{i} T_{k-1}^{i i} \partial_{i} \Gamma_{11}^{i} \bar{u}_{i i} \\
& +\bar{u}_{11}^{2} \sum_{i} T_{k-1}^{i i}+C \bar{u}_{11} .
\end{aligned}
$$

We estimate the first two terms on the right hand side

$$
\begin{aligned}
& \sum_{i} T_{k-1}^{i i}\left(\partial_{1} \partial_{1} g^{i i} \bar{u}_{i i}\right)+2 \sum_{i} T_{k-1}^{i i} \partial_{i} \Gamma_{11}^{i} \bar{u}_{i i} \\
& =\sum_{i} T_{k-1}^{i i}\left(R_{1 i 1 i} \bar{u}_{i i}\right) \leq C \max _{i}\left|\bar{u}_{i i}\right| \sum_{i} T_{k-1}^{i i} .
\end{aligned}
$$

Since we are in the cone $\Gamma_{k}^{+}$, the trace is positive by Proposition 1, and since $\bar{u}_{11}$ is the largest eigenvalue, we have

$$
\left|\bar{u}_{i i}\right| \leq(n-1) \bar{u}_{11}, \quad i=1 \ldots n .
$$

Therefore we obtain

$$
C+C \bar{u}_{11}+C \sum_{i} T_{k-1}^{i i}+C \bar{u}_{11} \sum_{i} T_{k-1}^{i i} \geq \bar{u}_{11}^{2} \sum_{i} T_{k-1}^{i i} .
$$

Dividing by $\bar{u}_{11}^{2}$ and using (4.15), we obtain

$$
\sigma_{k-1} \leq\left(\frac{C}{\bar{u}_{11}^{2}}+\frac{C}{\bar{u}_{11}}\right) \sigma_{k-1}+\frac{C}{\bar{u}_{11}^{2}}+\frac{C}{\bar{u}_{11}} .
$$

If

$$
\frac{C}{\bar{u}_{11}^{2}}+\frac{C}{\bar{u}_{11}} \geq \frac{1}{2}
$$

then we have the necessary eigenvalue bound. So we may assume that

$$
\frac{C}{\bar{u}_{11}^{2}}+\frac{C}{\bar{u}_{11}} \leq \frac{1}{2}
$$

and substitution into inequality (5.9) yields

$$
\frac{1}{2} \sigma_{k-1} \leq \frac{C}{\bar{u}_{11}^{2}}+\frac{C}{\bar{u}_{11}}
$$


Without loss of generality we may assume that $\bar{u}_{11} \geq 1$, and from the above inequality we obtain

$$
\sigma_{k-1} \leq C,
$$

which by Proposition 7 yields the eigenvalue bound in the case $k \geq 2$. In the case $k=1,(5.9)$ already gives the eigenvalue estimate.

\section{Existence.}

We now prove Theorem 1 . The main tool will be the degree theory for fully nonlinear second order elliptic equations as developed in [Li89]. We consider for $t \in[0,1]$ the family of equations

$$
t \sigma_{k}^{1 / k}+(1-t) \sigma_{1}=t \psi(x, u)+(1-t) \sigma_{1}(S) e^{u},
$$

where we abbreviate $\sigma_{k}^{1 / k}=\sigma_{k}^{1 / k}\left(\bar{\nabla}^{2} u\right)$. Note that at $t=0$, the equation is

$$
\Delta u+\frac{2-n}{2}|\nabla u|^{2}+\sigma_{1}(S)=\sigma_{1}(S) e^{u} .
$$

From the maximum principle, $u=0$ is the unique solution.

Proposition 9. For any $t \in[0,1]$, any $C^{2}$ solution $u^{t}$ of (6.1) with $\underline{\delta} \leq u \leq$ $\bar{\delta}$ satifies $\underline{\delta}<u<\bar{\delta}$.

Proof. From assumption (1.3), we have

$$
\begin{aligned}
t \psi(x, \underline{\delta})+(1-t) \sigma_{1}(S) e^{\underline{\delta}} & <t \sigma_{k}^{1 / k}(S)+(1-t) \sigma_{1}(S) \\
& <t \psi(x, \bar{\delta})+(1-t) \sigma_{1}(S) e^{\bar{\delta}},
\end{aligned}
$$

therefore the proof of Proposition 4 applies.

Proposition 10. Let $t \in[0,1]$, and $u^{t}$ be a solution to (6.1) with $\underline{\delta}<u^{t}<\bar{\delta}$. Then

$$
\left\|u^{t}\right\|_{C^{2}}<C
$$

for some constant $C$ independent of $t$.

Proof. We let $f_{t}=t \sigma_{k}^{1 / k}+(1-t) \sigma_{1}$. Define

$\Gamma_{k, t}^{+} \equiv$ component of $\left\{t \sigma_{k}^{1 / k}+(1-t) \sigma_{1}>0\right\}$ containing the positive cone. 
Then all of the estimates in the previous sections hold with $\sigma_{k}^{1 / k}$ replaced by $f_{t}$, and $\Gamma_{k}^{+}$replaced by $\Gamma_{k, t}^{+}$, and it is then not difficult to see that we can choose $C$ independent of $t$, since the $C^{0}$ estimate holds uniformly.

The above estimate yields uniform ellipticity, and since our equation is convex with respect to the second derivative variables, by the work of Evans [Eva82], and Krylov [Kry83] mentioned in the introduction, and standard elliptic theory, there exists a constant $M$ independent of $t$ such that

$$
\left\|u^{t}\right\|_{C^{4, \alpha}}<M
$$

Define the subset $\mathcal{O}_{t}$ of $C^{4, \alpha}$ by

$$
\begin{aligned}
\mathcal{O}_{t} \equiv & \left\{\underline{\delta}<u^{t}<\bar{\delta}\right\} \cap\left\{\left\|u^{t}\right\|_{C^{4, \alpha}}<M\right\} \\
& \cap\left\{\bar{\nabla}^{2} u^{t} \in \Gamma_{k, t}^{+}\right\} \cap\left\{t \sigma_{k}^{1 / k}+(1-t) \sigma_{1}>t \delta_{0}+(1-t) \sigma_{1}(S) e^{\underline{\delta}}\right\},
\end{aligned}
$$

where $\delta_{0}$ is a constant chosen such that $\psi(x, s)>\delta_{0}$ for $\underline{\delta}<s<\bar{\delta}$. Define $F_{t}: C^{4, \alpha} \rightarrow C^{2, \alpha}$ by

$$
F_{t}(u)=t \sigma_{k}^{1 / k}\left(\bar{\nabla}^{2} u\right)+(1-t) \sigma_{1}\left(\bar{\nabla}^{2} u\right)-t \psi(x, u)-(1-t) \sigma_{1}(S) e^{u} .
$$

There are no solutions of the equation $F_{t}(u)=0$ on $\partial \mathcal{O}_{t}$, so the degree of $F_{t}$ is well-defined and independent of $t$. As mentioned above, there is a unique solution at $t=0$. Furthermore, the linearization at $u=0$ is invertible. Therefore

$$
\operatorname{deg}\left(F_{0}, \mathcal{O}_{0}, 0\right)= \pm 1
$$

and since the degree is independent of $t$, we have

$$
\operatorname{deg}\left(F_{1}, \mathcal{O}_{1}, 0\right)= \pm 1
$$

and we conclude that (1.1) has a solution in $\mathcal{O}_{1}$.

Note that in the case $\psi(x, u)=f(x) e^{u}$, we can avoid using degree theory since the linearization is invertible, and the existence follows by using the continuity method.

\section{The negative cone equation.}

As mentioned in the introduction, the negative cone case of (1:1) is equivalent to the positive cone case of equation (1.4). We no longer necessarily 
have ellipticity along the straight line path for this equation (the proof of Proposition 3 does not work for this equation), so we just consider the equation

$$
\sigma_{k}^{1 / k}\left(\nabla^{2} u-d u \otimes d u+\frac{|\nabla u|^{2}}{2} g+S\right)=f(x) e^{u}>0 .
$$

In this section we will show that we still have the $C^{0}$ and $C^{1}$ estimate for solutions of this equation. The proof Proposition 5 still works for this equation, so we have

Proposition 11. Suppose $S \in C^{0}$ satisfies (1.2). Then there exist constants $\underline{\delta}<0<\bar{\delta}$ depending only upon $f$ and $S$, such that for any solution $u(x)$ of (7.1), we have $\underline{\delta}<u(x)<\bar{\delta}$.

The $C^{1}$ estimate also holds, with appropriate modifications to the proof of Proposition 6.

Proposition 12. Suppose $S \in C^{1},(1.2)$ is satisfied, and $u$ is a $C^{3}$ solution of (7.1) satisfying $\underline{\delta} \leq u(x) \leq \bar{\delta}$. Then there exists a constant $C_{1}$ depending only upon $S, \psi, \underline{\delta}, \bar{\delta}$, and $k$ such that

$$
|\nabla u|_{C^{0}} \leq C_{1} .
$$

Proof. We consider the following function

$$
h=\left(1+\frac{|\nabla u|^{2}}{2}\right) e^{\phi(u)}
$$

where $\phi: \mathbf{R} \rightarrow \mathbf{R}$ is a function of the form

$$
\phi(s)=c_{1}\left(c_{2}+s\right)^{p} .
$$

The proof procedes exactly as before, but we end up with the following analogue of equation (4.10)

$$
\begin{aligned}
& -\psi^{k-1}\left(\frac{u_{m}}{v} \psi_{m}+\psi_{u} \frac{|\nabla u|^{2}}{v}\right)-k \phi^{\prime}(u) \psi(x, u)^{k} \\
& \geq\left(\phi^{\prime \prime}(u)-\phi^{\prime}(u)^{2}-\phi^{\prime}(u)\right) T_{k-1}^{i j} u_{i} u_{j} \\
& \quad+T_{k-1}^{i j}\left(R_{i l j m} \frac{u_{l} u_{m}}{v}+\phi^{\prime}(u) \frac{|\nabla u|^{2}}{2} \delta_{i j}-\phi^{\prime}(u) S_{i j}+\frac{u_{l}}{v} \partial_{l} S_{i j}\right) .
\end{aligned}
$$


Lemma 4. Assume that $\underline{\delta}<s<\bar{\delta}$. Then we may choose constants $c_{1}, c_{2}$, and $p$ depending only upon $\underline{\delta}$, and $\bar{\delta}$. so that $\phi(s)=c_{1}\left(c_{2}+s\right)^{p}$ satisfies

$$
\phi^{\prime}(s)>0
$$

and

$$
\phi^{\prime \prime}(s)-\phi^{\prime}(s)^{2}-\phi^{\prime}(s)>0 .
$$

Proof. This follows easily from Proposition 3.

With $\phi(s)$ chosen as above, we let

$$
\epsilon_{1}=\min \left\{\phi^{\prime}(s)\right\}
$$

and

$$
\epsilon_{2}=\min \left\{\phi^{\prime \prime}(s)-\phi^{\prime}(s)^{2}+\phi^{\prime}(s)\right\} .
$$

From the inequality (7.2), we have

$$
C \geq \epsilon_{2} T_{k-1}^{i j} u_{i} u_{j}+T_{k-1}^{i j}\left(R_{i l j m} \frac{u_{l} u_{m}}{v}+\epsilon_{1} \frac{|\nabla u|^{2}}{2} \delta_{i j}+\phi^{\prime}(u) S_{i j}+\frac{u_{l}}{v} \partial_{l} S_{i j}\right) .
$$

The proof then procedes exactly as before.

We note that our method above for obtaining the $C^{2}$ estimate fails for equation (7.1), since the dominating term in the inequality (5.8) now has the wrong sign.

\section{Monge-Ampère equation in conformal geometry.}

In this section we restrict our attention to $k=n$, the determinant, and we consider more generally:

$$
\operatorname{det}^{1 / n}\left(\nabla^{2} u+d u \otimes d u-\frac{|\nabla u|^{2}}{2} g+S\right)=e^{-2 u},
$$

where $S \in \Gamma_{n}^{+}$is a positive definite symmetric tensor. 


\subsection{Proof of Theorem 2.}

We begin by proving a Harnack inequality for solutions of (8.1).

Proposition 13. Let $u$ be a $C^{2}$ solution of (8.1). If $\lambda_{\max }(S) D^{2}<\frac{\pi^{2}}{2}$, then

$$
2 \log \left(\cos \left(D \sqrt{\lambda_{\max }(S) / 2}\right)\right)+\sup u<\inf u,
$$

where $\lambda_{\max }(S)$ denotes the maximum eigenvalue of $S$ on $N$ and $D$ is the diameter.

Proof. In order to prove this, it is convenient to write the equation (8.1) in slighty different form. Writing $e^{u}=v^{2}$, with $v>0$, we see that $v$ solves the equation

$$
\operatorname{det}^{1 / n}\left(v \nabla^{2} v+d v \otimes d v-|\nabla v|^{2} g+\frac{1}{2} v^{2} S\right)=\frac{v^{-2}}{2},
$$

As seen in Section 2, we must have

$$
v \nabla^{2} v+d v \otimes d v-|\nabla v|^{2} g+\frac{1}{2} v^{2} S \in \Gamma_{n}^{+}
$$

and therefore since $v>0$,

$$
\nabla^{2} v+\frac{1}{2} v S \in \Gamma_{n}^{+}
$$

Next choose $p \in N$ such that $v(p)=\sup v$ and $q \in N$ such that $v(q)=\inf v$. Let $\gamma:[0, d(p, q)] \rightarrow N$ be a unit speed minimal geodesic such that $\gamma(0)=p$ and $\gamma(d(p, q))=q$. Letting $\bar{v}$ denote the restriction of $v$ to $\gamma$, we have

$$
\bar{v}^{\prime \prime}(t)+\frac{1}{2} S(\dot{\gamma}(t), \dot{\gamma}(t)) \bar{v}(t)>0
$$

therefore

$$
\bar{v}^{\prime \prime}(t)+\frac{1}{2} \lambda_{\max }(S) \bar{v}(t)>0,
$$

Let $M=v(p)=\sup v$, and $\alpha=\lambda_{\max }(S) / 2$. Then $w(t)=M \cos (\sqrt{\alpha} \cdot t)$ satisfies

$$
w^{\prime \prime}(t)+\alpha w(t)=0, w(0)=M=\bar{v}(0), w^{\prime}(0)=0=\bar{v}^{\prime}(0) .
$$


If we let $h(t)=(\bar{v} / w)(t)$, then it is easy to verify that $h$ satisfies the inequality

$$
h^{\prime \prime}>2 \sqrt{\alpha} \tan (\sqrt{\alpha} \cdot t) h^{\prime},
$$

for $\sqrt{\alpha} \cdot t<\pi / 2$. Integrating this, and using the boundary condition $h^{\prime}(0)=$ 0 , we find that $h^{\prime}(t)>0$ for $t>0$. Since $h(0)=1$, we conclude that $\bar{v}(t)>w(t)$ as long as $0<\sqrt{\alpha} \cdot t<\pi / 2$. Evaluating this at the endpoint $q$, we have

$$
v(q)=\inf v>\sup v \cdot \cos (\sqrt{\alpha} \cdot d(p, q)) \geq \sup v \cdot \cos (\sqrt{\alpha} \cdot D),
$$

that is,

$$
\sup v<\left(\cos \left(D \sqrt{\lambda_{\max }(S) / 2}\right)\right)^{-1} \inf v,
$$

Which implies the stated inequality for $u$.

Proposition 14. Let $u$ be a solution of (8.1), then there exist constants $\underline{\delta} \leq \bar{\delta}$ depending only upon $g, S$ so that $\sup u>\underline{\delta}$, and $\inf u<\underline{\delta}$.

Proof. This follows from the proof of Proposition 5, but since we have $e^{-2 u}$ instead of $e^{u}$, the inequalities are reversed.

Combining Propositions 13 and 14, we obtain the $C^{0}$ estimate:

Theorem 3. Let $u$ be a solution of (8.1). If

$$
\lambda_{\max }(S) D^{2}<\frac{\pi^{2}}{2},
$$

then there exist a constant $C$ depending only upon $g, S$ so that $|u| \leq C$.

Next, using this a priori estimate, we give a fixed point argument to prove the existence of a solution to 8.1.

Lemma 5. If $S \in \Gamma_{n}^{+}$satisfies (8.6), and $0<f(x) \in C^{\infty}(N)$ then the equation

$$
\operatorname{det}^{1 / n}\left(\nabla^{2} u+d u \otimes d u-\frac{|\nabla u|^{2}}{2} g+S\right)=f(x) e^{-\langle u\rangle}
$$

admits a unique solution $u \in C^{\infty}(N)$ where $\langle u\rangle=\int_{N} u d v o l_{g}$. 
Proof. We use the continuity method. For $t \in[0,1]$ we consider the equation

$$
F_{t}\left(u_{t}\right)=\operatorname{det}\left(\bar{\nabla}^{2} u_{t}\right)-f(x)^{n t} e^{-n\left\langle u_{t}\right\rangle},
$$

where

$$
\bar{\nabla}^{2} u_{t} \equiv \nabla^{2} u_{t}+d u_{t} \otimes d u_{t}-\frac{\left|\nabla u_{t}\right|^{2}}{2} g+(1-t) \lambda_{\max }(S) g+t S .
$$

Letting $A_{t}^{2, \alpha}(N)=\left\{u \in C^{2, \alpha}(N): \bar{\nabla}^{2} u_{t} \in \Gamma_{n}^{+}\right\}$, we know from Section 2 that a solution necessarily lies in $A_{t}^{2, \alpha}(N)$, and we claim that the map $F_{t}: A_{t}^{2, \alpha}(N) \rightarrow C^{\alpha}(N)$ is locally invertible at a solution. From (2.7) above we see that the linearized operator is

$$
F_{t}^{\prime}\left(u_{t}\right)(h)=T_{n-1}\left(\bar{\nabla}^{2} u_{t}\right)^{i j}\left(\nabla_{\tilde{g}_{t}}^{2} h\right)_{i j}+n f(x)^{n t} e^{-n\left\langle u_{t}\right\rangle}\langle h\rangle,
$$

where $\tilde{g}_{t}=e^{-2 u_{t}} g$. The coefficient matrix $T_{n-1}\left(\bar{\nabla}^{2} u_{t}\right)$ is positive definite, but there is a slight difficulty due to the fact that the linearized operator is not formally self-adjoint. Nevertheless, it is still invertible. This was proved for Monge-Ampère equations in [Del81], and the proof given there is applicable in this case. Local invertibility of $F_{t}$ follows from the implicit function theorem (see [GT83]).

Let $u_{t} \in C^{2, \alpha}(N)$ be a solution of (8.8). The matrix $S_{t} \equiv(1-$ t) $\lambda_{\max }(S) g+t S$ satifies the condition (8.6) for all $t \in[0,1]$, therefore we have that $u_{t}$ satisfies the Harnack inequality (8.2). Let $q \in N$ be a point where $u_{t}$ attains a global minimum. We have

$$
\operatorname{det}^{1 / n}\left(S_{t}\right) \leq f(q) e^{-\left\langle u_{t}\right\rangle},
$$

which implies $\left\langle u_{t}\right\rangle<C$. By also considering a maximum of $u_{t}$, we obtain the estimate $\left|\left\langle u_{t}\right\rangle\right| \leq C$. Combining this with the Harnack inequality, we obtain an a priori $L^{\infty}$ estimate on $u_{t}$, independent of $t$. From the work in Sections 4 and 5, and Evans-Krylov, we obtain an a priori bound on the $C^{2, \alpha}$ norm of $u_{t}$, independent of $t$ for some $\alpha \in(0,1)$. Standard elliptic theory gives a uniform bound on the $C^{k, \alpha}$ norm for each $k \geq 3$.

We consider the equation $F_{0}\left(u_{0}\right)=0$ :

$$
\operatorname{det}^{1 / n}\left(\nabla^{2} u_{0}+d u_{0} \otimes d u_{0}-\frac{\left|\nabla u_{0}\right|^{2}}{2} g+\lambda_{\max }(S) g\right)=e^{-\left\langle u_{0}\right\rangle} .
$$

Let $u_{0}$ be any solution to (8.12). As before, by going to a maximum and minimum of $u_{0}$, we find that $e^{-\left\langle u_{0}\right\rangle}=\lambda_{\max }(S)$. Then from the arithmeticgeometric inequality, we have

$$
\lambda_{\max }(S)=e^{-\left\langle u_{0}\right\rangle} \leq \frac{1}{n} \Delta u_{0}+\frac{2-n}{2 n}\left|\nabla u_{0}\right|^{2}+\lambda_{\max }(S) .
$$


We conclude that $\Delta u_{0} \geq 0$, which implies $u_{0}=$ constant. The existence of a solution at $t=1$ now follows from the continuity method.

It remains to prove the uniqueness at $t=1$. To see this, if we have 2 distinct solutions $u_{1}$ and $v_{1}$ at $t=1$, we may run the continuity method in reverse. From uniqueness at $t=0$, the paths we obtain must hit at some time $t_{0} \in[0,1)$. But since the linearization is invertible at $t_{0}$, this contradicts local invertibility.

Theorem 4. If $A_{g} \in \Gamma_{n}^{+}$satisfies $\lambda_{\max }\left(A_{g}\right) D^{2}<\frac{\pi^{2}}{2}$ then the equation

$$
\operatorname{det}^{1 / n}\left(\nabla^{2} u+d u \otimes d u-\frac{|\nabla u|^{2}}{2} g+A_{g}\right)=e^{-2 u}
$$

admits a solution $u \in C^{\infty}(N)$.

Proof. We will employ a fixed point argument using the existence and uniqueness of solutions to (8.7) in Lemma 5. For $t \in[0,1], \alpha \in(0,1)$, and $u \in C^{2, \alpha}(N)$, let $u_{t}=H(u, t)$ denote the unique solution in $C^{2, \alpha}(N)$ of the equation:

$$
\begin{aligned}
& \operatorname{det}^{1 / n}\left(\nabla^{2} u_{t}+d u_{t} \otimes d u_{t}-\frac{\left|\nabla u_{t}\right|^{2}}{2} g+t A_{g}+(1-t) \lambda_{\max }\left(A_{g}\right) g\right) \\
& =e^{-2 t u} e^{-\left\langle u_{t}\right\rangle}
\end{aligned}
$$

It is easy to show that for each $u \in C^{2, \alpha}(N)$, the mapping $H(u, t):[0,1] \rightarrow$ $C^{2, \alpha}(N)$ is uniformly continuous in $t$, and we also claim that for each $t \in$ $[0,1], H(u, t): C^{2, \alpha}(N) \rightarrow C^{2, \alpha}(N)$ is a compact operator. For a bounded subset of $C^{2, \alpha}(N)$, the right hand side is bounded in $C^{2, \alpha}(N)$. From the proof of Lemma 5, solutions are bounded in $C^{3, \alpha}(N)$. Since $C^{3, \alpha} \subset C^{2, \alpha}$ is a compact embedding, the claim follows.

We next show that for all $t \in[0,1]$, solutions of the equation $u=H(u, t)$ satisfy an a priori bound $\|u\|_{C^{2, \alpha}(N)}<C$. As in the proof of Lemma 5, we need only obtain an $L^{\infty}$ estimate.

To this end, let $u_{t} \in C^{2, \alpha}(N)$ be a fixed point $H\left(u_{t}, t\right)=u_{t}$, and $q \in N$ be a point where $u_{t}$ attains a global minimum. Then we have at $q$,

$$
\operatorname{det}^{1 / n}\left(A_{g}(q)\right) \leq e^{-\left\langle u_{t}\right\rangle} e^{-2 t u_{t}(q)},
$$

which implies

$$
C_{1} \leq-\left\langle u_{t}\right\rangle-2 t \inf u_{t}
$$


for some constant $C_{1}$, and we obtain the estimate

$$
(\operatorname{Vol}(N)+2 t) \inf u_{t} \leq C_{1} .
$$

Similary by considering a maximum of $u_{t}$ we obtain

$$
(\operatorname{Vol}(N)+2 t) \sup u_{t} \geq C_{2},
$$

for some constant $C_{2}$. These estimates, coupled with the Harnack inequality in Proposition 8.2, imply the desired uniform $L^{\infty}$ estimate.

As already seen in the proof of Lemma 5 , we have that $H(u, 0) \equiv C$ for all $u \in C^{2, \alpha}$, where $C$ is some constant. We may then apply a fixed point theorem of Berger [Ber77, Theorem 5.4.14, p. 270]:

Proposition 15. Let $H(x, t)$ be a one-parameter family of compact operators defined on a Banach space $X$ for $t \in[0,1]$, with $H(x, t)$ uniformly continuous in $t$ for fixed $x \in X$. Furthermore, suppose that every solution of $x=H(x, t)$ for some $t \in[0,1]$, is contained in the fixed open ball $\sigma=\{x \mid\|x\|<M\}$. Then, assuming $H(x, 0) \equiv 0$, the compact operator $H(x, 1)$ has a fixed point $x \in \Sigma$.

Letting $X=C^{2, \alpha}(N)$, we find a fixed point $u \in C^{2, \alpha}(N)$ at $t=1$. Standard regularity theory then implies that $u \in C^{\infty}(N)$. Adding a constant if necessary, we obtain a solution to (8.13).

To finish the proof of Theorem 2, if $\sigma([g])<\frac{\pi^{2}}{2}$, then there exists a metric $\bar{g} \in[g]$ with $\lambda_{\max }\left(A_{\bar{g}}\right) D^{2}<\frac{\pi^{2}}{2}$. The existence of a conformal metric $\tilde{g}$ with $\operatorname{det}\left(A_{\tilde{g}}\right)=1$ follows from Theorem 4, and the compactness of the space of such solutions was also demonstrated in the proof of Theorem 4.

\subsection{Examples.}

In this section we examine some simple cases, and we refer the reader to [Pet98] for details.

- $\left(S^{n}, g=\right.$ round metric $): R i c=(n-1) g, D=\pi$, and

$$
\lambda_{\max }\left(A_{g}\right) D^{2}=\pi^{2} / 2 \text {. }
$$

If $\sigma\left(S^{n}, g\right)<\frac{\pi^{2}}{2}$, then Theorem 2 would imply that the space of solutions of (1.8) is compact. But compactness cannot hold in this case since $S^{n}$ has a non-compact group of conformal transformations, and the orbit of 
the standard metric gives rise to a non-compact family of solutions of (1.8). Therefore $\sigma\left(S^{n}, g\right)=\frac{\pi^{2}}{2}$.

- $\left(R P^{n}, g=\right.$ standard metric) : Ric $=(n-1) g, D=\pi / 2$, and

$$
\lambda_{\max }\left(A_{g}\right) D^{2}=\pi^{2} / 8<\pi^{2} / 2 .
$$

From [Via00b], we know that the standard metric on $R P^{n}$ is the unique solution in its conformal class of (1.8), but this shows that conformal classes on $R P^{n}$ in a large neighborhood of the standard metric have compactness.

- $\left(C P^{m}, g=\right.$ Fubini-Study $): R i c=(2 m+2) g, D=\pi / 2$, and

$$
\lambda_{\max }\left(A_{g}\right) D^{2}=\frac{m+1}{2 m-1} \frac{\pi^{2}}{4}<\pi^{2} / 2 .
$$

In this case, we do not know if the Fubini-Study metric is the unique solution in its conformal class to (1.8) since it is not locally conformally flat, but the above shows that the space of solutions is compact, and also for conformal classes on $C P^{m}$ in a large neighborhood of Fubini-Study.

\section{References.}

[Ber77] M.S. Berger, Nonlinearity and functional analysis, Academic Press [Harcourt Brace Jovanovich Publishers], New York, 1977, Lectures on nonlinear problems in mathematical analysis, Pure and Applied Mathematics.

[Bes87] A.L. Besse, Einstein manifolds, Springer-Verlag, Berlin, 1987.

[CGY01] A. Chang, M. Gursky, and P. Yang, An a priori estimate for a fully nonlinear equation on four-manifolds, preprint, 2001.

[CNS85] L. Caffarelli, L. Nirenberg, and J. Spruck, The Dirichlet problem for nonlinear second-order elliptic equations. III. Functions of the eigenvalues of the Hessian, Acta Math., 155(3-4) (1985), 261-301.

[Del81] P. Delanoë, Équations du type de Monge-Ampère sur les variétés riemanniennes compactes, II, J. Funct. Anal., 41(3) (1981), 341353.

[Eva82] L.C. Evans, Classical solutions of fully nonlinear, convex, secondorder elliptic equations, Comm. Pure Appl. Math., 35(3) (1982), 333-363. 
[Gär59] L. Gärding, An inequality for hyperbolic polynomials, J. Math. Mech., 8 (1959), 957-965.

[GT83] D. Gilbarg and N.S. Trudinger, Elliptic partial differential equations of second order, second ed., Springer-Verlag, Berlin, 1983.

[Kry83] N.V. Krylov, Boundedly inhomogeneous elliptic and parabolic equations in a domain, Izv. Akad. Nauk SSSR Ser. Mat., 47(1) (1983), 75-108.

[Li89] Y.Y. Li, Degree theory for second order nonlinear elliptic operators and its applications, Comm. Partial Differential Equations, 14(11) (1989), 1541-1578.

[Li90] Y.Y. Li, Some existence results for fully nonlinear elliptic equations of Monge-Ampère type, Comm. Pure Appl. Math., 43(2) (1990), 233-271.

[LP87] J.M. Lee and T.H. Parker, The Yamabe problem, Bull. Amer. Math. Soc. (N.S.), 17(1) (1987), 37-91.

[Pet98] P. Petersen, Riemannian geometry, Springer-Verlag, New York, 1998.

[Rei73] R.C. Reilly, On the Hessian of a function and the curvatures of its graph, Michigan Math. J., 20 (1973), 373-383.

[Sch89] R.M. Schoen, Variational theory for the total scalar curvature functional for Riemannian metrics and related topics, Topics in calculus of variations (Montecatini Terme, 1987), Lecture Notes in Math., 1365, Springer, Berlin, (1989), 120-154.

[Sch91] R.M. Schoen, On the number of constant scalar curvature metrics in a conformal class, Differential geometry, Longman Sci. Tech., Harlow, (1991), 311-320.

[Via00a] J.A. Viaclovsky, Conformal geometry, contact geometry, and the calculus of variations, Duke Math. J., 101(2) (2000), 283-316.

[Via00b] J.A. Viaclovsky, Conformally invariant Monge-Ampère equations: global solutions, Trans. Amer. Math. Soc., 352(9) (2000), 43714379 . 
[Via00c] J.A. Viaclovsky, Some fully nonlinear equations in conformal geometry, Differential equations and mathematical physics (Birmingham, AL, 1999), Amer. Math. Soc., Providence, RI, (2000), 425-433.

Department of MATHEMATiCS

Massachusetts Institute of TeChNology

CAMBridge, MA 02139

E-mail address: jeffv@math.mit.edu

RECeIved OCTOBER 12, 2000. 